\title{
SPONTANEOUS THROMBOSIS OF NON-TUBERCULOUS PULMONARY ARTERY MYCOTIC PSEUDOANEURYSM IN PROLONGED CHILDHOOD PNEUMONIA.
}

Idris Ibrahim ${ }^{1}$, Mohd Naim Mohd Yaakob ${ }^{1}$, Mohd Hazeman Zakaria ${ }^{1}$, Anas Tharek ${ }^{1}$, Nurul Syazwani Rahmat ${ }^{1}$, Mohd Naqib Mohd Sabri ${ }^{1}$, Mohd Fandi Al-Khafiz Kamis ${ }^{1}$.

${ }^{1}$ Department of Radiology, hospital pengajar universiti putra malaysia (HPUPM), 43400 Serdang, Selangor.

\section{*Corresponding author:}

Dr. Idris Ibrahim, Department of of Radiology, hospital pengajar universiti putra malaysia (HPUPM), 43400 Serdang, Selangor. Email: dr.idris.19121986@gmail.com

DOI: https://doi.org/10.32896/tij.v1n1.12-16

Published: 30.06 .2021

\begin{abstract}
Pulmonary tuberculosis (PTB) is common in tropical country like Malaysia. Prolonged PTB infection may lead to mycotic pulmonary artery pseudoaneurysm (PAP). We report a case of childhood nontuberculous pulmonary infection causing mycotic PAP which resolved spontaneously after antibiotics therapy.

A 1 year 6 months old girl underlying Down syndrome presented with prolonged fever for two weeks, cough and breathlessness. Her leucocytes count were elevated and she developed several hypotensive episodes secondary to septicaemia. Chest radiograph showed loculated right sided pleural effusion. Ultrasound revealed complex pleural collection and initial aspiration revealed a thick stale blood. Thinking of possible vascular cause, ultrasound able to locate a well-defined rounded structure with high flow velocity seen on Doppler ultrasound consistent with pseudoaneurysm and CT thorax confirmed the findings. Pulmonary artery angiogram prior to embolization revealed no evidence of abnormal vasculature or contrast blush at the region of interest. Complimentary ultrasound showed evidence of spontaneous thrombosis within the pseudoaneurysm.

Non-tuberculous PAP is a rare but possible life-threatening sequela of pneumonia. Pleural drainage in a haemothorax with concomitant mycotic thoracic pseudoaneurysm may cause loss of pressure tamponade and will end up with devastating consequences. Careful ultrasound image acquisition must be made by the attending radiologist prior to pleural drainage.
\end{abstract}

Keywords: Paediatric, Pulmonary artery pseudo-aneurysm, Pulmonary infection, Spontaneous thrombosis.

\section{INTRODUCTION}

The incidence of pulmonary artery pseudo aneurysm (PAP) secondary to infective causes is a rare occurrence in modern medicine due to the easily assessable antibiotics treatment. However, in some cases with prolonged or partially treated chest infection, high bacterial load may cause invasion of the adjacent pulmonary vasculature causing weakening of the vessel wall thus make it vulnerable to pseudoaneurysm formation. In tropical countries, the incidence of tuberculous mycotic pseudoaneurysm is common and is widely reported in the literature. We report a case of nontuberculous PAP secondary to prolonged pneumonia in a paediatric patient which undergone spontaneous thrombosis. 


\section{CASE REPORT}

A 1 year 6 months old girl with underlying Down syndrome, congenital hypothyroidism, small patent ductal arteriosus (PDA) and anorectal malformation presented on day 15 of illness with prolonged fever for two weeks and cough for 1 week. The fever started when she was previously admitted for stoma closure and pull through surgery for anorectal malformation. Initial chest Xray showed infective changes and she was treated with intravenous Imipenem and Metronidazole for one week. The symptoms were resolving upon discharge, and she completed a one-week course of oral antibiotics at home. There were episodes of temperature spikes at home, which were well controlled by anti-pyretic medicines. However, her cough worsens, and she started to develop breathlessness which eventually made the parents brought her to the emergency department.

Upon arrival at the emergency department, she is tachypnoeic with reduced air entry on the right. Blood analysis reveals leukocystosis with neutrophil predominance /elevated white cell count which was $21.7 \times 10^{9} / \mathrm{L}$ which shows neutrophil predominance of $81.5 \%$. Chest x-ray shows large loculated right pleural effusion (Figure 1). Due to the ongoing respiratory distress, ultrasound guided drainage of the pleural effusion was carried out to alleviate the symptoms. Initial ultrasound showed complex septated collection with hyperchoic/echogenic internal debris. An 18G Chiba needle was used as puncture needle and the first aspiration revealed a thick stale bloody fluid. The needle was retracted and a further careful examination of the loculated effusion was carried out and a well-defined rounded structure with internal high velocity flow Doppler signal resembling a pseudoaneurysm was found (Figure 4A). The previously noted hyperchoic/echogenic debris was actually blood products. An immediate contrasted CT thorax was done, and it confirms the diagnosis of pseudoaneurysm which was seen to arise from the descending branch of right pulmonary artery (Figure 2). There were also changes of active lung infection in the right lower lobe seen which includes cavitation, consolidation and ground glass lesions.

The child was subsequently admitted to the ICU due to worsening respiratory distress. Once her vitals were stable, a pulmonary artery angiogram was carried out with the intention to embolise the pseudoaneurysm. However, there was no contrast blush were found or evidence of pseudoaneurysm at the region of interest during angiogram. Complimentary ultrasound on the table showed presence of echo within the pseudoaneurysm with no Doppler flow signal detected suggestive of thrombosis (Figure 4B). In view of the patient's age and stable clinical parameters, repeated CT scan was not done to limit the radiation exposure. Two nasal swab cultures were done and came back positive for Chryseobacteriumindologenes and Elizabethkingiameningoseptica. The sputum and blood cultures as well as tuberculosis screening were all negative. She completed multiple intravenous antibiotic and antifungal courses including Ceftazidime, Gentamicin, Fluconazole and Metronidazole. The respiratory symptoms were finally subsided, and she was discharge home well. 


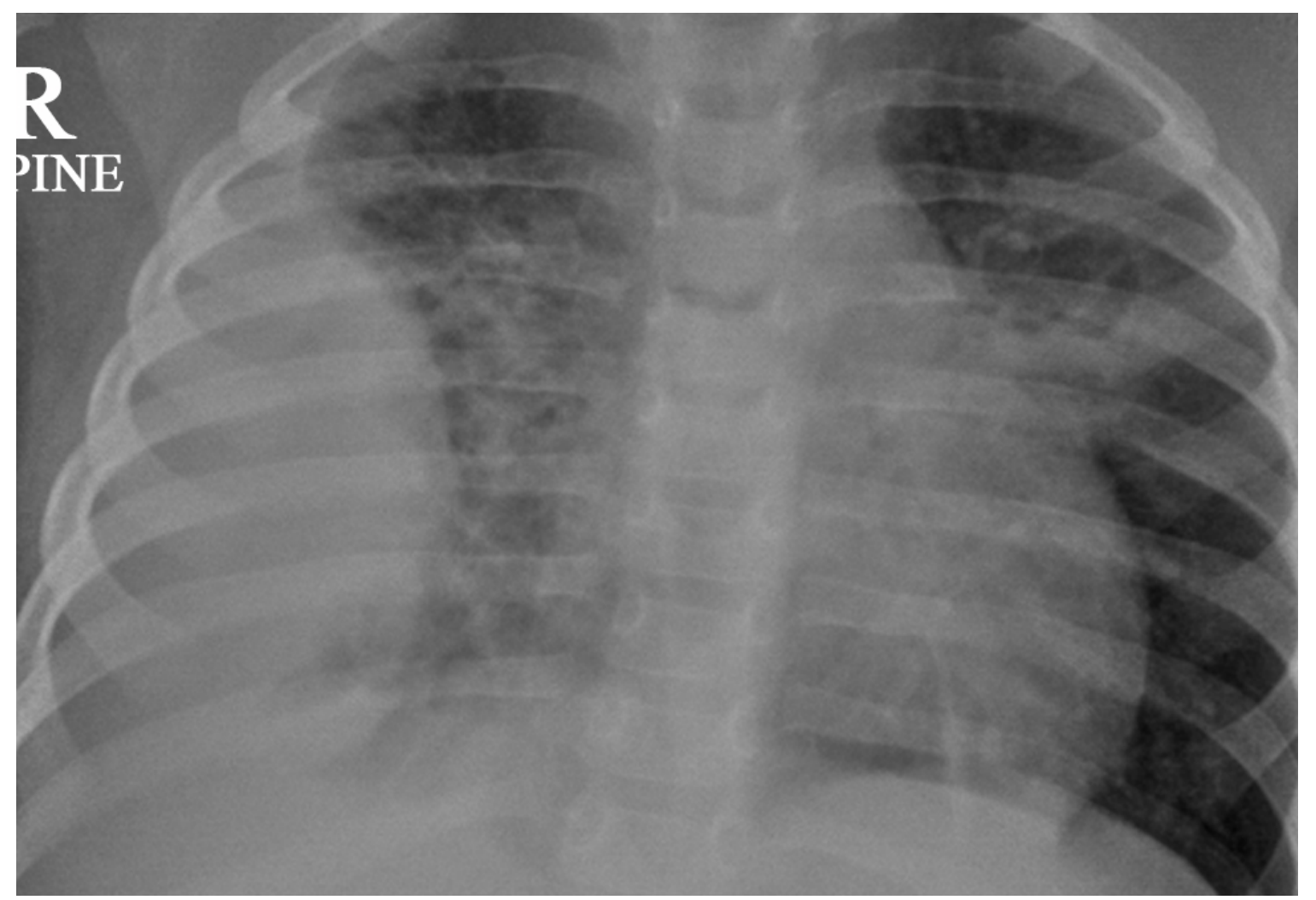

Figure 1: Initial chest x-ray upon presentation showed loculated right pleural effusion and infective lung changes.
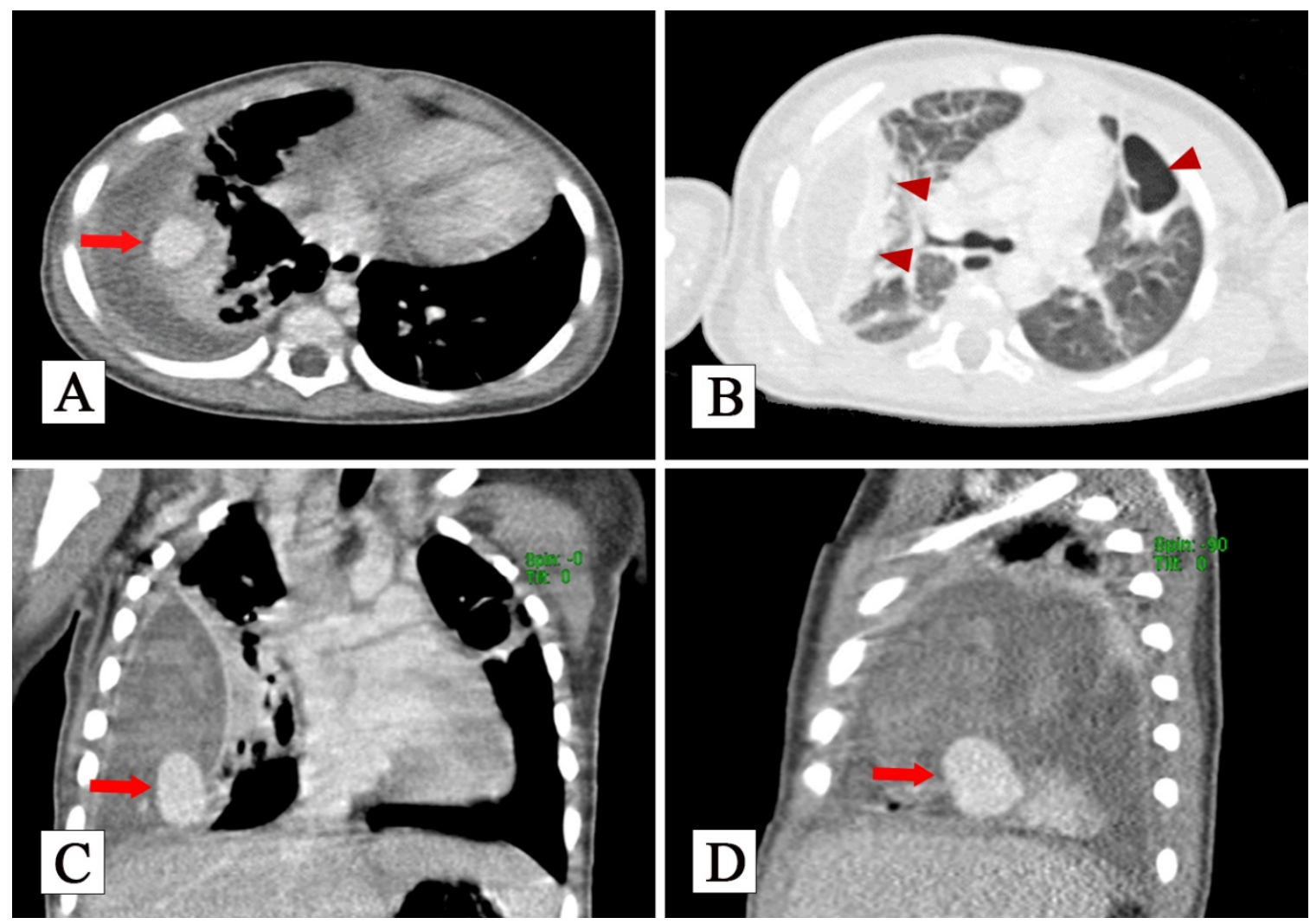

Figure 2: Contrast-enhanced CT thorax confirms presence of pseudoaneurysm arising from descending branch of right pulmonary artery (block arrow). Note the infective changes consists of consolidation, ground glass lesions and and cavitation. 


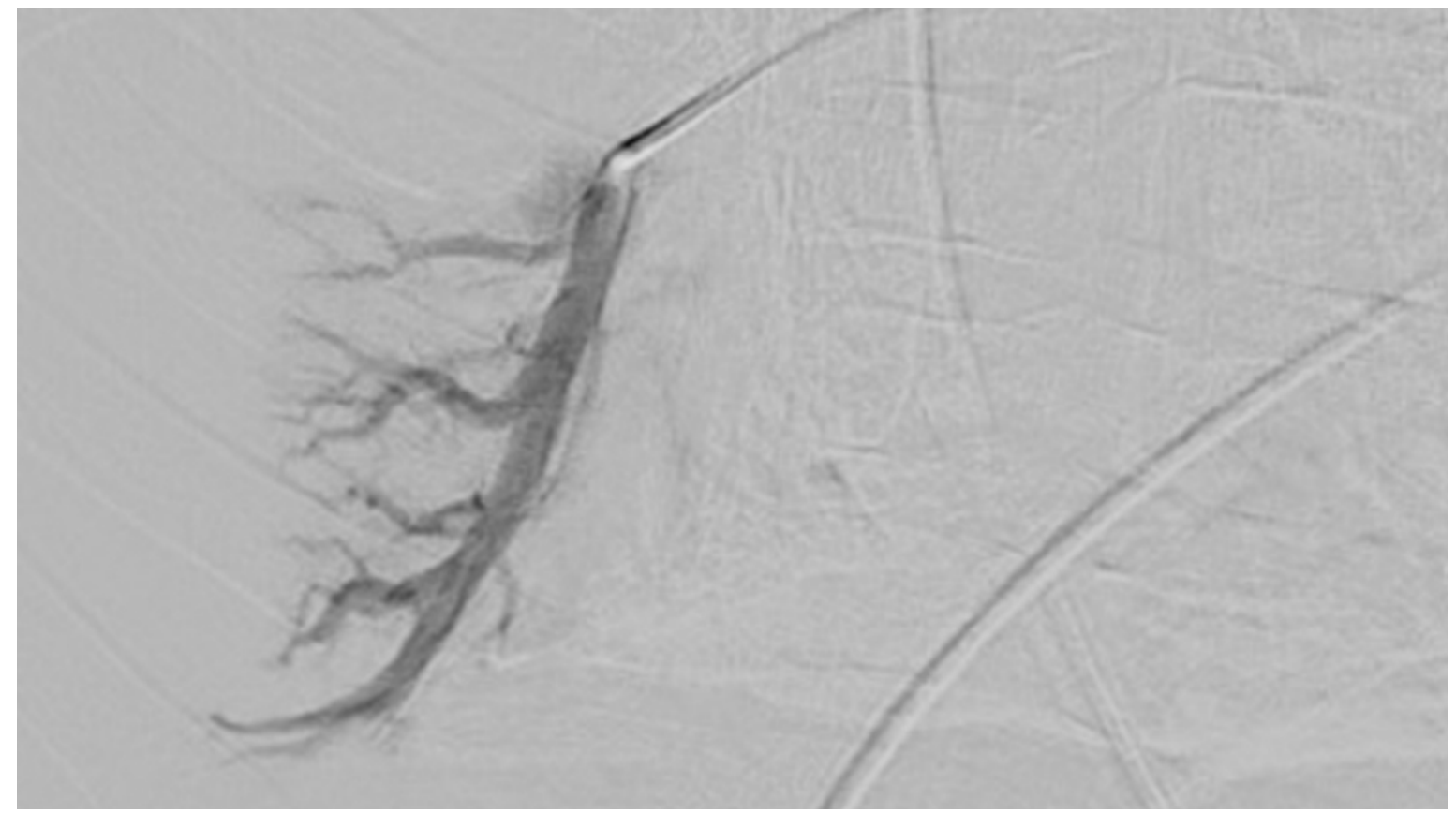

Figure3: Selective Digital Subtraction Angiogram of the descending branch of pulmonary artery. Note there is no evidenceof pseudoaneurysm or contrastextravasation.

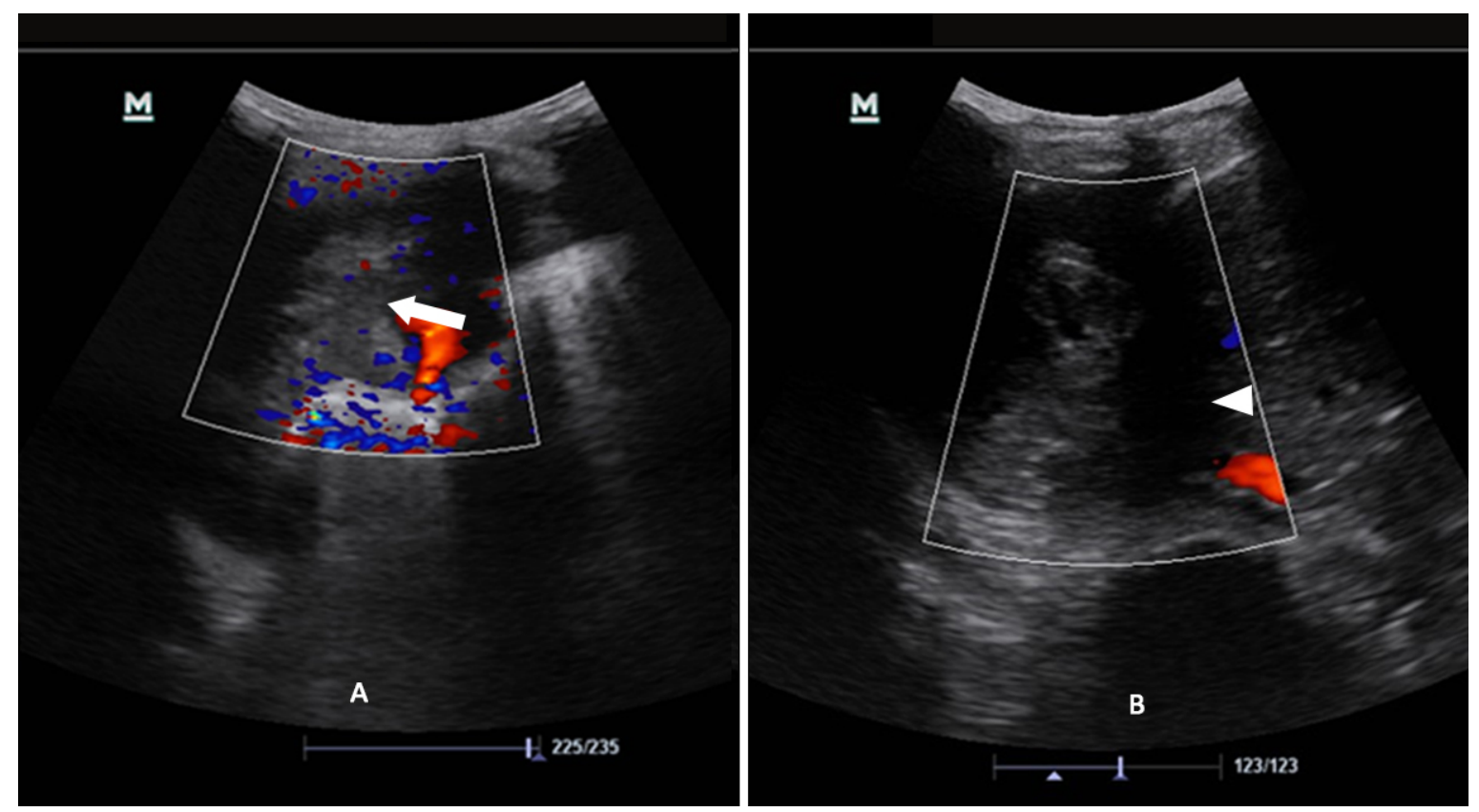

Figure 4A: Initial ultrasound showed rounded hypoechogenic structure with doppler flow in keeping with pseudoaneurysm (Blockarrow).

Figure 5B: On the table ultrasound during transcatheter angiogram noted spontaneous thrombosis within the previously seen pseudoaneurysm (Arrowhead). 


\section{DISCUSSION}

Pulmonary artery mycotic pseudoaneurysm (PAP) is one of the rare complications of prolonged childhood pneumonia. Pulmonary tuberculosis (TB) and syphilis were responsible for the majority of PAP with syphilis affecting the large pulmonary arteries and TB affecting smaller intraparenchymal vessels $(1,2)$. In chronic pulmonary $\mathrm{TB}$, the prevalence of pseudoaneurysm was reported to be around $4 \%$, and often associated with significant mortality rate due to massive bleeding (3). Besides tuberculosis, several literatures reported cases of PAP secondary to bacterial and viral pneumonia. Bacterial cause of PAP was previously reported in a patient with ventilator-associated necrotizing pneumonia who developed life-threatening haemoptysis and was treated with coil embolization (4). There was also a case of PAP arising from $\mathrm{H} 1 \mathrm{~N} 1$ Influenza viral pneumonia which was successfully treated with coiling as well (5). In the paediatric age group, there is previous report of mycotic PAP as a sequela of necrotizing pneumonia in 6 months old infant who presented with haemoptysis. This case demonstrated that coil embolization is a good treatment of choice in paediatric population. Follow up CT scan months later showed complete resolution of the aneurysm (6).

The first line therapeutic options mentioned in most of the literature are minimally invasive procedure such as bronchoscopy coagulation and

\section{REFERENCES}

1. Bartter T, Irwin RS, Nash G: Aneurysms of the pulmonary arteries. Chest 94:1065-1075, 1988

2. Carlos S. Restrepo, Aimee P. Carswell: Aneurysm and pseudoaneurysm of pulmonary vasculature, Semin Ultrasound CT MRI 33:552-566 (C) 2012 Elsevier

3. Auerbach O: Pathology and pathogenesis of pulmonary artery aneurysms in tuberculous cavity. Am Rev Tuberc 39:99-115, 1939

4. Michael Kalina, Frederick Giberson, hemoptysis secondary to pulmonary artery pseudoaneurysm after necrotizing pneumonia (2007) Ann ThoracSurg 2007;84:1386-7

5. Joseph C. Lee, Darren L. Walters, Richard E. Slaughter (2011), Angioembolisation of Pulmonary Artery Pseudoaneurysm Arising embolization. Endovascular treatment was proven to be safe and effective to seal off the PAP $(6,7)$. In cases of failed first line treatment, surgical ligation will be the next option. There is also previous case example of PAP secondary to trauma which recurs after surgical ligation and further treated with coil embolization (8). Although, most of the published cases of PAP are reported to be successfully managed by endovascular procedure or surgical resection, there is a slight chance of spontaneous resolution or thrombosis within the aneurysm. There is a reported case of Rasmussen's aneurysm which spontaneously resolved after nine months of anti-tuberculosis treatment (9).

\section{CONCLUSION}

In conclusion, non-tuberculous PAP is a rare but possible life-threatening sequela of pneumonia. An effort must be made by Radiologist to screen for any abnormal Doppler signal within thoracic collection to exclude these rare complications especially prior to therapeutic interventional procedures as drainage of haemothorax secondary to PAP may lead to devastating consequences due to loss of pressure tamponade. Trans-catheter embolization may be considered as first line treatment regardless the age of the patient or clinical presentation. Occasionally, pseudoaneurysm may resolve by spontaneous thrombosis similar in this case study.

in H1N1 Influenza Viral Pneumonia, Clinical spotlight, p599-601.

6. Hemanth D, Krantikumar R, Ashwin G, Rahul S Rupture mycotic pulmonary artery pseudoaneurysm in an infant: Transcatheter embolization and $\mathrm{CT}$ assessment.

7. GhayeB, Trottreu G, Dondellinger R.F, Multiple pulmonary artery psedoaneurysms: Intrasaccular embolization, Eur. Radiol. 7, 176-179 (1997).

8. Khan AA, Bauer TL, Garcia MJ, Panasuk, Davies AL. Angiographic embolization of a traumatic pulmonary pseudoaneurysm. Ann ThoracSurg2005;79:2136-8.

9. Kim HY, Song KS, Goo JM, Lee JS, Lee KS, Lim TH. Thoracic sequelae and complications of tuberculosis. Radiographics 21:839-860, 2001. 